\title{
Nasal Chondromesenchymal Hamartoma
}

National Cancer Institute

\section{Source}

National Cancer Institute. Nasal Chondromesenchymal Hamartoma. NCI Thesaurus. Code C122985.

A very rare benign lesion that arises from the sinonasal tract. It usually affects infants and children. It presents as a polypoid mass and is composed of cartilage, myxoid stroma, and other mesenchymal elements. 